\title{
Age-dependent Increase in Ortho-Tyrosine and Methionine Sulfoxide in Human Skin Collagen Is Not Accelerated in Diabetes

\author{
Evidence against a Generalized Increase in Oxidative Stress in Diabetes
}

\author{
Mary C. Wells-Knecht, ${ }^{\star}$ Timothy J. Lyons, ${ }^{\ddagger}$ David R. McCance, ${ }^{\S}$ Suzanne R. Thorpe, ${ }^{\star}$ and John W. Baynes*\| \\ *Department of Chemistry and Biochemistry, and $\|$ School of Medicine, University of South Carolina, Columbia, South Carolina 29208; \\ ${ }^{\ddagger}$ Department of Medicine, Medical University of South Carolina, Charleston, South Carolina 29425; and ${ }^{\S}$ Sir George E. Clark Metabolic \\ Unit, Royal Victoria Hospital, Belfast BT12 6BA, Northern Ireland
}

\begin{abstract}
The glycoxidation products $\mathrm{N}^{\epsilon}$-(carboxymethyl)lysine and pentosidine increase in skin collagen with age and at an accelerated rate in diabetes. Their age-adjusted concentrations in skin collagen are correlated with the severity of diabetic complications. To determine the relative roles of increased glycation and/or oxidation in the accelerated formation of glycoxidation products in diabetes, we measured levels of amino acid oxidation products, distinct from glycoxidative modifications of amino acids, as independent indicators of oxidative stress and damage to collagen in aging and diabetes. We show that ortho-tyrosine and methionine sulfoxide are formed in concert with $\mathrm{N}^{\epsilon}$-(carboxymethyl)lysine and pentosidine during glycoxidation of collagen in vitro, and that they also increase with age in human skin collagen. The ageadjusted levels of these oxidized amino acids in collagen was the same in diabetic and nondiabetic subjects, arguing that diabetes per se does not cause an increase in oxidative stress or damage to extracellular matrix proteins. These results provide evidence for an age-dependent increase in oxidative damage to collagen and support previous conclusions that the increase in glycoxidation products in skin collagen in diabetes can be explained by the increase in glycemia alone, without invoking a generalized, diabetes-dependent increase in oxidative stress. (J. Clin. Invest. 1997. 100:839846.) Key words: advanced glycation end-products • carboxymethyllysine $\cdot$ glycation $\bullet$ oxidation $\cdot$ pentosidine
\end{abstract}

\section{Introduction}

Advanced glycation end-products (AGEs), ${ }_{1}^{1}$ formed by nonenzymatic Maillard reactions between carbohydrates and proteins, contribute to the age-dependent increase in chemical

Address correspondence to Dr. John W. Baynes, Department of Chemistry and Biochemistry, University of South Carolina, Columbia, SC 29208. Phone: 803-777-7272; FAX: 803-777-9521; E-mail: baynes@psc.sc.edu

Received for publication 17 December 1996 and accepted in revised form 12 May 1997.

1. Abbreviations used in this paper: AGE, advanced glycation endproduct; CML, $\mathrm{N}^{\epsilon}$-(carboxymethyl)lysine; FL, N $\mathrm{N}^{\epsilon}$ (1-deoxy-D-fructos1 -yl)lysine (fructoselysine); MetSO, methionine sulfoxide; $o$-Tyr, ortho-tyrosine; ROS, reactive oxygen species.

J. Clin. Invest.

(c) The American Society for Clinical Investigation, Inc. 0021-9738/97/08/0839/08 \$2.00

Volume 100, Number 4, August 1997, 839-846

http://www.jci.org modification and cross-linking of long lived tissue proteins (1, 2 ). Increased glycation of proteins and formation of AGEs are also implicated in the pathogenesis of the long term complications of diabetes (3-5). $\mathrm{N}^{\epsilon}$-(carboxymethyl)lysine (CML) and pentosidine are frequently used chemical biomarkers of AGE formation in tissues, and both of these compounds increase in proteins with age in skin collagen and at an accelerated rate in diabetes $(6,7)$. Because they are formed in reactions of proteins with glucose only under oxidative conditions, they have been termed glycoxidation products (1). Based on the increase in age-adjusted levels of CML and pentosidine in diabetic collagen, Dyer et al. (7) concluded that diabetes may be considered, at least at the chemical level, as a disease characterized by accelerated aging of tissue collagens by advanced glycation and oxidation reactions. However, the relative importance of increased glycation and/or oxidation in the formation of AGEs in diabetes has not been rigorously evaluated.

Both blood glucose and protein glycation are increased and have been identified as potential sources of oxidative stress (i.e., increased production of reactive oxidant species [ROS]) in diabetes $(8,9)$. Thus, hyperglycemia may simultaneously enhance both glycative and oxidative stress, and the increase in glycation and oxidation chemistry may contribute synergistically to the formation of AGEs and development of diabetic complications (10). Still, despite growing evidence of increased oxidative stress in diabetes (11), Dyer et al. (7) concluded that the age-corrected levels of glycoxidation products in skin collagen of diabetic patients could be explained solely by the duration of diabetes and the long term increase in blood glucose concentration, as measured by glycation of hemoglobin or collagen, without invoking an increase in oxidative stress in diabetes. However, their conclusion is based on the assumption that AGEs are derived only from glucose or glycated proteins and is compromised by uncertainties regarding the actual origin of glycoxidation products. Thus, not only glucose but also ascorbate, pentoses, and other carbohydrates may form both CML and pentosidine $(12,13)$, and recent work indicates that CML may also be formed from products of lipid peroxidation, independent of glycoxidation reactions (14).

To obtain an independent assessment of the status of oxidative stress in diabetes, we set out to determine if non-carbohydrate-derived amino acid oxidation products were formed during glycoxidation of protein in vitro and, if so, whether the levels of these products were also increased in human skin collagen from diabetic compared with age-matched nondiabetic individuals. We reasoned that age-adjusted levels of oxidized amino acids would provide an index of oxidative stress and damage to extracellular protein, structurally distinct from glycoxidative modification of amino acids. The two products on which we focused represent a range of susceptibility of amino acids to oxidation: methionine sulfoxide (MetSO), which is formed readily and in significant yield under mild oxidizing con- 
ditions $(15,16)$, and ortho-tyrosine (o-Tyr), which is formed in low yield during metal-catalyzed and radiolytic oxidation of proteins $(17,18)$. We show here that both MetSO and $o$-Tyr are formed in concert with CML and pentosidine during glycation of collagen under oxidative conditions in vitro. We also show that both MetSO and $o$-Tyr increase normally with age in human skin collagen, providing evidence of an age-dependent increase in oxidative damage to collagen, independent of formation of glycoxidation products. However, levels of MetSO and $o$-Tyr were not increased in skin collagen from diabetic compared with nondiabetic subjects, arguing that there is not a systemic increase in oxidative stress in the extracellular matrix in diabetes.

\section{Methods}

Materials. Reagents were purchased from Sigma Chemical Co. (St. Louis, MO) or Aldrich Chemical Co. (Milwaukee, WI) unless otherwise indicated. Internal standards $\left[{ }^{13} \mathrm{C}_{6}\right]$-fructoselysine (FL) and $\left[{ }^{2} \mathrm{H}_{8}\right]$-CML were prepared as described previously (19). $\left[{ }^{13} \mathrm{C}_{6}\right]$-Phe $(99$ atom\%) was purchased from Cambridge Isotope Laboratories (Woburn, MA), and $\left[{ }^{13} \mathrm{C}_{6}\right]$-o-Tyr was synthesized from $\left[{ }^{13} \mathrm{C}_{6}\right]$-Phe as described previously for $\left[{ }^{2} \mathrm{H}_{4}\right]-o$-Tyr (15).

In vitro incubations. Rat tail collagen was prepared from $150-200 \mathrm{~g}$ rats and incubated with glucose in $200 \mathrm{mM}$ phosphate buffer, $\mathrm{pH} 7.4$, under oxidative or antioxidative conditions at $37^{\circ} \mathrm{C}$, as described previously $(20,21)$. Oxidative incubations were conducted under aerobic conditions, and antioxidative incubations under a nitrogen (anaerobic) atmosphere in buffer containing 1-mM concentrations of diethylenetriaminepentaacetic acid and phytic acid to chelate transition metal catalysts of oxidation chemistry. To completely inhibit oxidative chemistry during long term antioxidative reactions, the solutions were thoroughly degassed under vacuum, replenished with nitrogen, and then incubated in a tissue culture incubator under nitrogen. Otherwise, methionine sulfoxide, a sensitive indicator of oxidative chemistry was sometimes detected in antioxidative reaction mixtures. Individual vials were set up for each time point and frozen at $-20^{\circ} \mathrm{C}$ until analysis.

Patient samples. Human skin collagen samples were obtained from nondiabetic and diabetic donors or at autopsy at the Royal Victoria Hospital. The protocol was approved by the Institutional Review Board for use of Human Subjects. Samples were processed as described previously (7) and stored at $-70^{\circ} \mathrm{C}$ until analysis. Measurements of $o$-Tyr in human skin collagen were performed using samples from 23 subjects without diabetes, aged $0-85 \mathrm{yr}$, and 17 subjects with insulin-dependent diabetes mellitus, aged 17-58 yr. Duration of diabetes was $14.4 \pm 10.7 \mathrm{yr}$. Glycated hemoglobin (percent $\mathrm{HbA}_{1}$ ), measured by agar gel electrophoresis (Corning Medical, Halsted, UK) was 10.4 \pm 1.8 ; the percent $\mathrm{HbA}_{1}$ range for a nondiabetic reference population was $3.6-7.2 \%$. Because of limited amounts of samples, measurements of MetSO were performed on a subset of 17 subjects without diabetes, aged 2-85 yr, and 12 subjects with insulin-dependent diabetes mellitus, aged 19-58 yr. For this group, duration of diabetes was $14.8-11.2 \mathrm{yr}$, and percent $\mathrm{HbA}_{1}$ was 10.7-1.6.

Gas chromatography-mass spectrometry analyses. Electron impact ionization-selected ion monitoring-gas chromatography-mass spectrometry analyses were performed on a 5890 gas chromatograph-5970 mass selective detector system (Hewlett-Packard Co., Palo Alto, CA) (7), with the exception of $o$-Tyr in human skin collagen (see below), which was performed on a double focusing magnetic sector mass spectrometer (VG 70SQ; Fisons Instruments, Altrincham, UK). All analyses were performed using a $30-\mathrm{m} \mathrm{Rtx}^{\circledR}-5$ capillary column (Restek Corp., Bellefonte, PA). Peak areas of each analyte were normalized to their respective, heavy labeled internal standard, and quantitation was based on calibration curves generated using increasing amounts of each analyte and constant amounts of internal standards.
To normalize for the amount of protein analyzed, final values of glycation, glycoxidation, and oxidation products were expressed as a ratio to their precursor amino acids, Lys, $o$-Tyr or Met, respectively.

Measurement of FL, CML, and pentosidine. These compounds were analyzed after hydrolysis of collagen, as described previously (22). FL was measured as the N,O-trifluoroacetyl trifluoroethyl ester derivative of its hydrolysis product, furosine. CML was measured in $\mathrm{NaBH}_{4}$-reduced samples as the $\mathrm{N}$-trifluoroacetyl trifluoroethyl ester derivative. Pentosidine was measured in $\mathrm{NaBH}_{4}$-reduced samples by reverse phase-HPLC with fluorescence detection $(\mathrm{Ex}=328 \mathrm{~nm} ; \mathrm{Em}=$ $378 \mathrm{~nm})$.

Measurement of o-Tyr. Internal standards for $o$-Tyr and Phe $\left(0.05 \mathrm{nmol}\left[{ }^{13} \mathrm{C}_{6}\right]-o\right.$-Tyr and $130 \mathrm{nmol}\left[{ }^{13} \mathrm{C}_{6}\right]$-Phe $)$ were added to unreduced samples before hydrolysis. After hydrolysis under $\mathrm{N}_{2}$ in $6 \mathrm{~N}$ $\mathrm{HCl}$ for $24 \mathrm{~h}$ at $110^{\circ} \mathrm{C}$, Phe and $o$-Tyr were measured by selected ion monitoring-gas chromatography-mass spectrometry as their acetyl isopropyl ester derivatives, as described previously (15). For rat tail collagen samples, the 265 and 271 ions were monitored for $o$-Tyr and $\left[{ }^{13} \mathrm{C}_{6}\right]-o$-Tyr, respectively, and the 249 and 255 ions were monitored for Phe and $\left[{ }^{13} \mathrm{C}_{6}\right]$-Phe, respectively. To increase sensitivity and specificity for measurement of $o$-Tyr in human skin collagen, these samples were analyzed by high resolution mass spectrometry, monitoring the 265.1314 and 271.1515 ions for $o$-Tyr and $\left[{ }^{13} \mathrm{C}_{6}\right]-o$-Tyr, respectively. Because of the sample requirement for measurement of $o$-Tyr, the signal from the $\mathrm{M}^{+}$ions of $\mathrm{Phe}$ and ${ }^{13} \mathrm{C}_{6}$-Phe $(249.1365$ and 255.1566 , respectively) exceeded the dynamic range of the data system. Therefore, the natural abundance $\left[{ }^{13} \mathrm{C}\right]$ ions of the $\mathrm{M}^{+}$ions of Phe and $\left[{ }^{13} \mathrm{C}_{6}\right]$-Phe (250.1398 and 256.1600 , respectively) were monitored for measurement of Phe, resulting in an $85-90 \%$ reduction in peak areas. The following temperature program was used: 2 min at $100^{\circ} \mathrm{C}$, ramp at $5^{\circ} \mathrm{C} / \mathrm{min}$ to $260^{\circ} \mathrm{C}$, ramp at $15^{\circ} \mathrm{C} / \mathrm{min}$ to $290^{\circ} \mathrm{C}$, hold at $290^{\circ} \mathrm{C}$ for $3 \mathrm{~min}$.

Measurement of MetSO. MetSO was measured as methionine after two sequential cyanogen bromide treatments of unreduced collagen (21). Samples were hydrolyzed as above, except that dithiothreitol ( $5 \mathrm{mM})$ was included to ensure complete conversion of MetSO to Met during hydrolysis. Met, homoserine, and homoserine lactone were measured by amino acid analysis using cation exchange chromatography and postcolumn fluorescence detection with $o$-phthaldialdehyde (23). The MetSO content of collagen is expressed relative to its total Met + MetSO content.

Data analysis. Statistical analyses were performed by the Spearman Rank Correlation method for nonparametric data using SigmaStat (Jandel Scientific, San Rafael, CA), and by Spearman-Rank Partial Correlations using SAS ${ }^{\circledR}$ (SAS Institute Inc., Cary, NC) to correct for correlation with age. A value of $P \leq 0.05$ was judged statistically significant.

\section{Results}

Kinetics of formation of glycation, glycoxidation and oxidation products during glycation of collagen in vitro. Collagen was incubated for various times (0-5 wk) in the presence of 250 $\mathrm{mM}$ glucose in $200 \mathrm{mM}$ phosphate buffer, $\mathrm{pH} 7.4$, at $37^{\circ} \mathrm{C}$ under oxidative or antioxidative conditions. The Amadori adduct FL was measured to determine the extent of glycation of the protein. CML and pentosidine were measured as indicators of glycoxidation, and MetSO and $o$-Tyr as indices of oxidative damage to the amino acid backbone of the protein, as well as overall oxidative stress in the reaction system. The kinetics of formation of FL, CML, and pentosidine were consistent with those of previous studies $(20,21)$. Thus, initial rates of formation of FL (Fig. $1 A$ ) were comparable under oxidative and antioxidative conditions, but higher yields of FL were obtained at 3-5 wk under antioxidative conditions. Recent work suggests that these differences result from the more rapid rate of degra- 


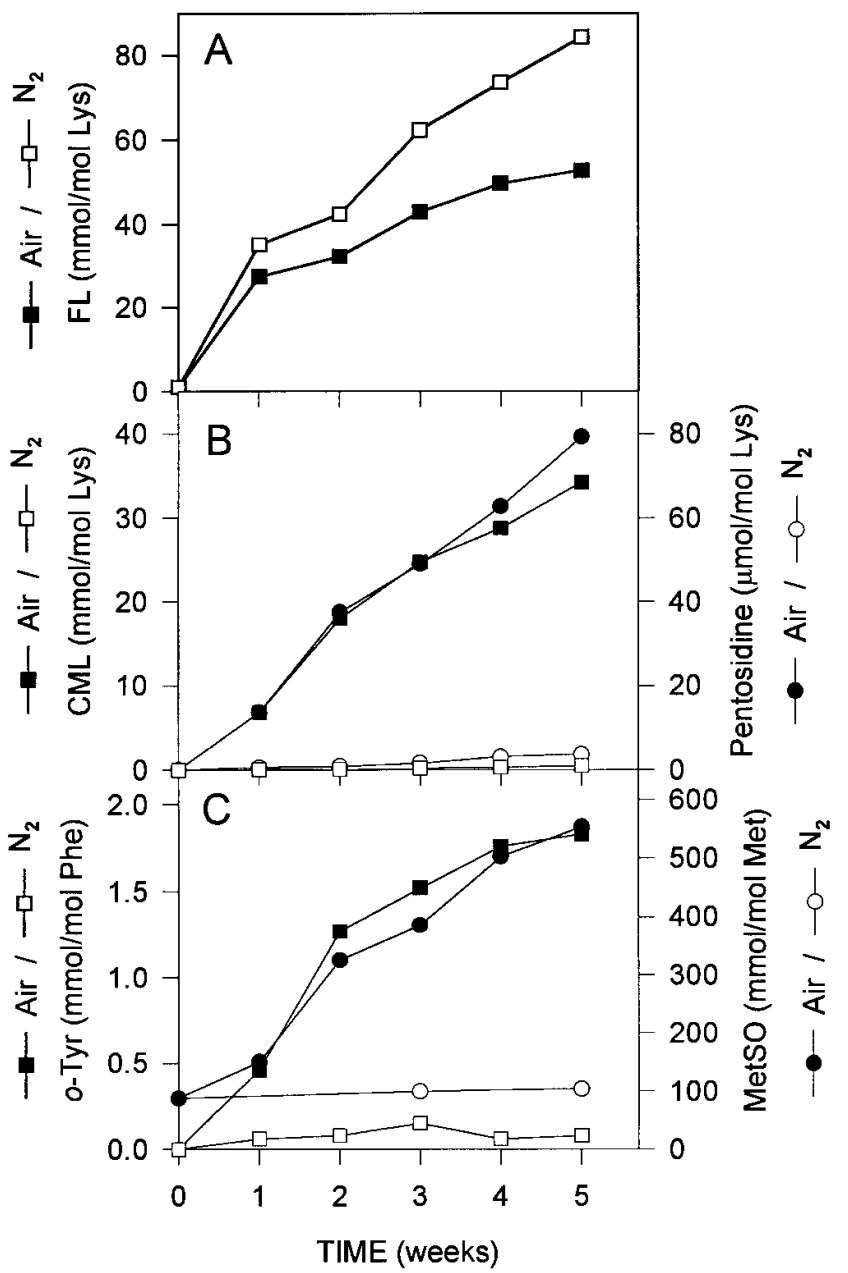

Figure 1. Kinetics of formation of $(A)$ fructoselysine, $(B) \mathrm{CML}$ and pentosidine, and $(C)$ MetSO and $o$-Tyr during glycation of collagen in vitro under oxidative and antioxidative conditions. Collagen was incubated in the presence of $250 \mathrm{mM}$ glucose in $200 \mathrm{mM}$ phosphate buffer, $\mathrm{pH} 7.4$, at $37^{\circ} \mathrm{C}$ under oxidative or antioxidative conditions. Samples were removed at the indicated times for analysis. Results shown in this figure are from a single experiment, representative of at least three independent studies and measurements. Note that the vertical axes of the various plots differ significantly in scale.

dation of FL to CML and other products under oxidative conditions (24). CML and pentosidine were formed only under oxidative conditions, but at significantly different rates, yielding a $\mathrm{CML} /$ pentosidine concentration ratio of $\sim 50$ at $5 \mathrm{wk}$ (Fig. 1 B). For comparison, the relative CML/pentosidine concentrations in human skin collagen are $\sim 40$ (7).

Like the glycoxidation products, MetSO and $o$-Tyr were formed only under oxidative conditions, and their concentrations increased with time of incubation of collagen with glucose (Fig. $1 C$ ). At $5 \mathrm{wk}, o$-Tyr accounted for $\sim 0.2 \%$ of the Phe content of the collagen. MetSO was a more sensitive indicator of oxidative damage, accounting for $>50 \%$ of the original Met in collagen at $5 \mathrm{wk}$. A background level of endogenous MetSO was also detected in the starting rat tail collagen, as in human skin collagen (see below). The rate of formation of MetSO was $\sim 300 \times$ that of $o$-Tyr (Fig. $1 C$ ).

To determine the effect of glucose concentration on the formation of MetSO and $o$-Tyr, collagen was incubated for $5 \mathrm{wk}$ with various concentrations of glucose under oxidative or antioxidative conditions. The glucose concentration was varied from normoglycemic $(5 \mathrm{mM})$ and hyperglycemic $(25 \mathrm{mM})$ concentrations to higher concentrations commonly employed in in vitro model systems to accelerate AGE formation. As observed previously $(19,20)$, the yield of FL increased with glucose concentration and was $25-30 \%$ higher under antioxidative compared with oxidative conditions (Fig. $2 A$ ). Glycoxidation products (Fig. $2 B$ ) and amino acid oxidation products (Fig. $2 C$ ) were formed only under oxidative conditions. Neither glycoxidation products nor MetSO or $o$-Tyr increased during a 5-wk incubation under antioxidative conditions in the absence of glucose. (Compare levels of oxidation products in sample at zero time in Fig. $1 C$ with 5 -wk incubation at zero glucose concentration in Fig. $2 C$ ). Effects of glucose concen-

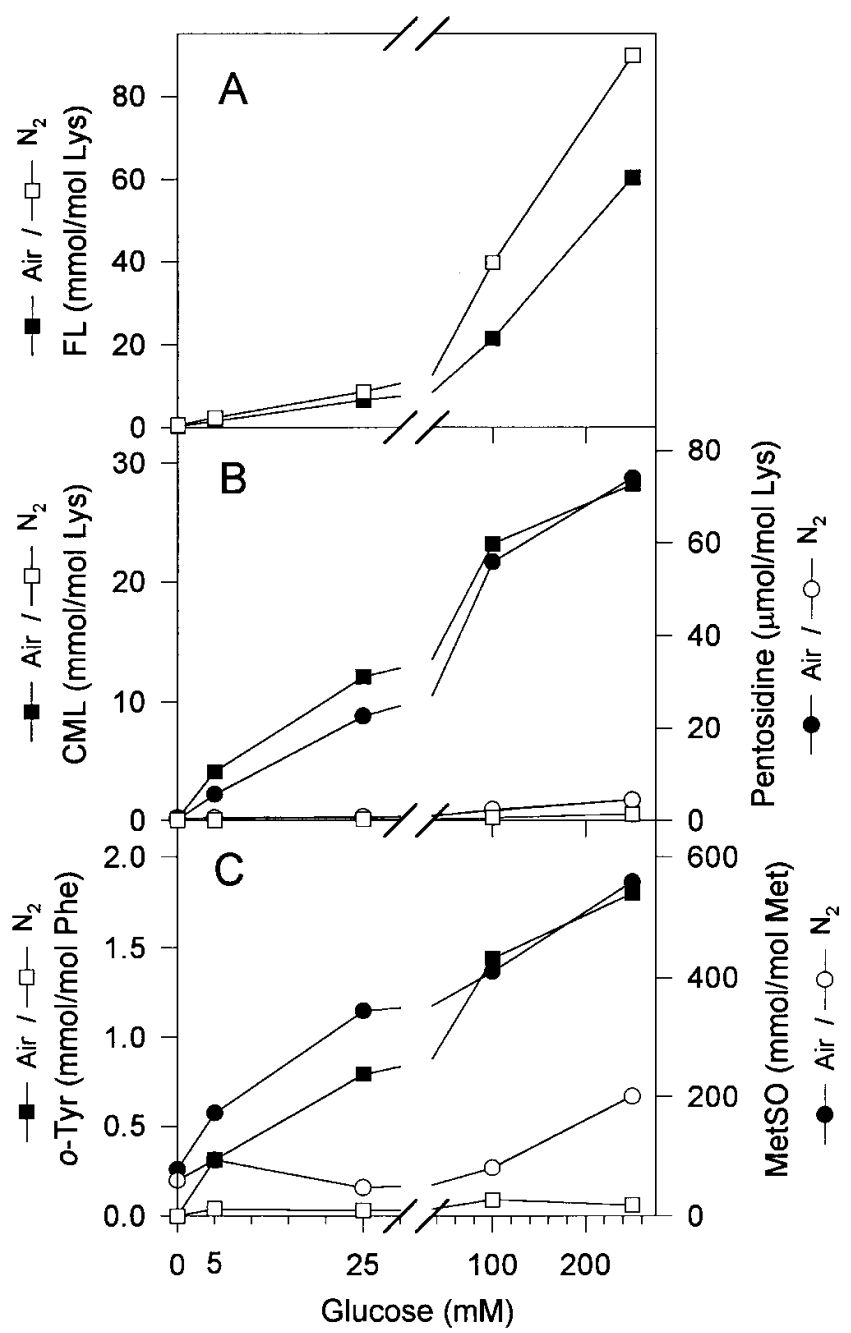

Figure 2. Effect of glucose concentration on formation of $(A)$ fructoselysine, $(B) \mathrm{CML}$ and pentosidine, and $(C) \mathrm{MetSO}$ and $o$-Tyr during glycation of collagen in vitro under oxidative and antioxidative conditions. Collagen was incubated with indicated concentrations of glucose under conditions described in Fig. 1 and analyzed after reaction for $5 \mathrm{wk}$. Results shown in this figure are from a single experiment, representative of at least three independent studies and measurements. Note that the vertical axes of the various plots differ significantly in scale. 
tration on formation of MetSO and $o$-Tyr closely paralleled effects on formation of glycoxidation products in these incubations, including the trend to a plateau at higher glucose concentrations. This plateau might result from inhibition of metalcatalyzed autoxidation reactions by the antioxidant and metal chelating activities of products formed during the late stages of the Maillard reaction (25). The ratios of CML/pentosidine and $\mathrm{MetSO} / o$-Tyr were relatively independent of glucose concentration and similar to those observed in Fig. 1. Notably, both MetSO and $o$-Tyr were readily detected in collagen incubated with $5 \mathrm{mM}$ glucose and increased two- to threefold in collagen incubated in $25 \mathrm{mM}$ glucose, suggesting that they should be useful as carbohydrate-independent biomarkers for assessing the status of oxidative stress in vivo under hyperglycemic compared with normoglycemic conditions. As shown below, however, although levels of MetSO and $o$-Tyr increased with age in skin collagen, their age-adjusted levels were not increased in diabetes, suggesting that increased oxidative stress does not contribute to the increase in glycoxidation of collagen in diabetes.

Measurement of MetSO and o-Tyr in human skin collagen. As shown in Fig. 3, there was a strong correlation between both the MetSO and $o$-Tyr content of skin collagen and donor age for nondiabetic subjects. Linear least squares analysis of the line for the nondiabetic population (Fig. 3) indicated that MetSO and $o$-Tyr increased approximately three- to fourfold in skin collagen between ages 10 and $80 \mathrm{yr}$. In previous studies

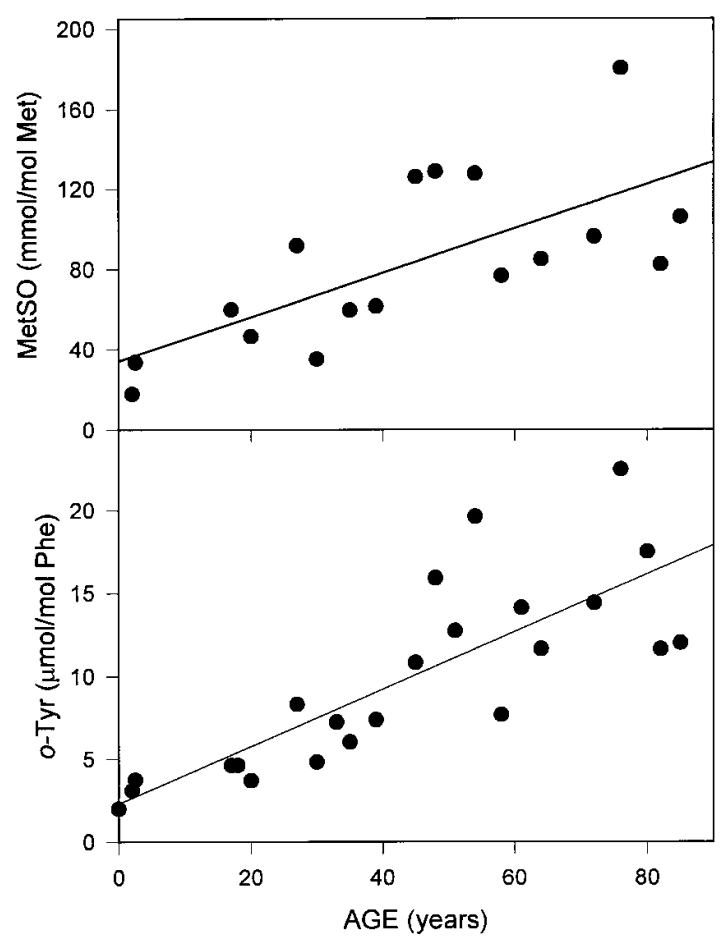

Figure 3. Concentrations of MetSO and $o$-Tyr in human skin collagen as a function of age. MetSO $(t o p)$. The equation of the linear regression line is: $\mathrm{mmol} \mathrm{MetSO} / \mathrm{mol} \mathrm{Met}=1.1 \times$ age $+34(n=17$, $r=0.72, P=0.001)$. $o$-Tyr (bottom). The equation of the linear regression line is: $\mu \mathrm{mol} o-\mathrm{Tyr} / \mathrm{mol} \mathrm{Phe}=0.17 \times$ age $+2.3(n=23, r=$ $0.85, P<0.001)$. Lines drawn are linear least-squares fit to the data. Statistical analyses were performed by Spearman Rank Correlation.

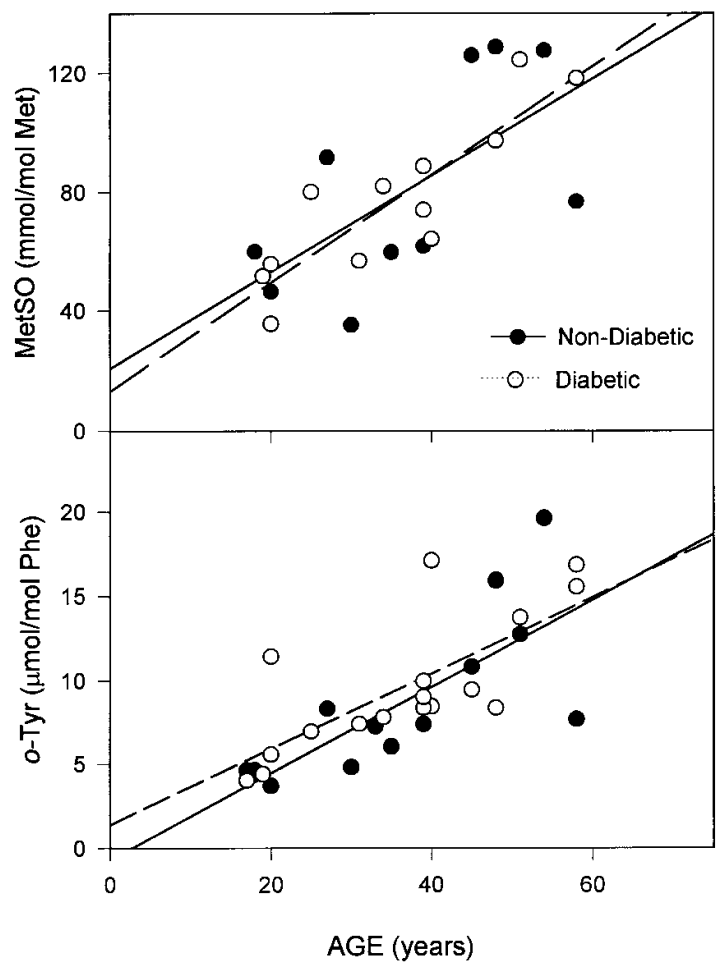

Figure 4. Age-dependent increase in MetSO and $o$-Tyr in human skin collagen from diabetic and nondiabetic subjects. MetSO (top). The equation of the line for the nondiabetic samples (solid line and symbols) is: $\mathrm{mmol} \mathrm{MetSO} / \mathrm{mol} \mathrm{Met}=1.6 \times$ age $+20.7(n=10$, $r=0.67, P=0.04)$; the equation of the line for the diabetic samples (dashed line, $\bigcirc)$ is $\mathrm{mmol} \mathrm{MetSO} / \mathrm{mol} \mathrm{Met}=1.8 \times$ age $+13.2(n=12$, $r=0.86, P<0.001)$. $o$-Tyr (bottom). The equation of the line for the nondiabetic samples (solid line, $\bullet$ ) is $\mu \mathrm{mol} o$-Tyr $/ \mathrm{mol} \mathrm{Phe}=0.88 \times$ age $+1.2(n=13, r=0.81, P<0.001)$; the equation of the line for the diabetic samples (dashed line, $O$ ) is $\mathrm{mmol} o$-Tyr $/ \mathrm{mol} \mathrm{Phe}=$ $0.85 \times$ age $+4.6(n=17, r=0.76, P<0.001)$. The lines drawn are linear least squares fits to the data. Statistical analyses were performed by Spearman Rank Correlation.

(7), we had observed approximately sevenfold increases in CML and pentosidine during the same age range. As shown in Fig. 4, there were no differences in levels of MetSO between the diabetic and control groups (Fig. $4 A$, two-sided $P=0.92$ for combined sample size of 22) nor in levels of $o$-Tyr in skin collagen from a group of diabetic patients, compared with agematched control subjects (Fig. $4 B$, two-sided $P=0.36$ for the combined sample size of 30). The magnitude of the $P$ values and the lack of significance for both MetSO and $o$-Tyr support the conclusion that there is no difference in age-adjusted levels of MetSO and $o$-Tyr in skin collagen from diabetic compared with control subjects. Simulated $t$ and $P$ values for double and triple the sample sizes were also calculated by sample size increase simulation using the current trend and variance and were also nonsignificant, supporting the strength of the conclusions. As shown in Fig. 5, the levels of MetSO and $o$-Tyr also correlated strongly with one another in both groups. For both diabetic and control subjects, levels of MetSO in human skin collagen were $\sim 6,000-8,000 \times$ higher than those of $o$-Tyr, significantly greater than the ratios observed in collagen glycoxidized in vitro $(\sim 300$; Figs. $1 C$ and $2 C$ ). The difference in ra- 


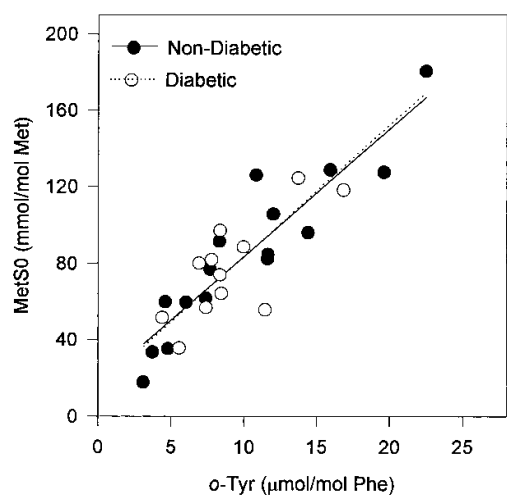

Figure 5. Comparison of concentrations of MetSO and $o$-Tyr in human skin collagen from nondiabetic $(\bigcirc)$ and diabetic $(\bullet)$ subjects.

The lines drawn are linear least squares fits to the data for the nondiabetic (solid line) and diabetic (dashed line) samples. Statistical analyses by Spearman Rank Correlation were nondiabetic, $n=16$,

$r=0.94, P<0.001$; diabetic, $n=12, r=0.64, P=0.025$; total group, $n=30, r=0.86, P<0.001$.

tios between the in vivo and in vitro systems probably results from differences in the nature or relative concentrations of ROS in the two systems.

The concentrations of the various oxidation and glycoxidation products in collagen correlated with one another in both the diabetic and nondiabetic populations, with $r$ values ranging from $0.68-0.85$ and $P$ values ranging from 0.008 to $<0.0001$ for the various comparisons (Table I). Partial correlation analyses, correcting for the effect of age, are summarized in Table II. In this case, for comparison with the diabetic samples, correlations were calculated for both the total group of nondiabetic samples and separately for those nondiabetic samples that spanned the same age range as the diabetic samples. After correction for effects of age, there were still significant partial correlations of MetSO and $o$-Tyr with both CML and pentosidine in the nondiabetic population, but not in the diabetic population. Overall, there were strong correlations between oxidation and glycoxidation products in human skin collagen, and age-corrected partial correlations remained significant in the nondiabetic group, but not in the diabetic group.

\section{Discussion}

Oxidative stress in diabetes. In this study, we have demonstrated that two biomarkers of oxidative stress and damage to protein, MetSO and $o$-Tyr, increase in concert with the glycoxidation products, CML and pentosidine, during glycation of collagen under oxidative conditions in vitro. These results document that oxidative damage to amino acids in proteins occurs during glycoxidation reactions, probably as a result of superoxide and $\mathrm{H}_{2} \mathrm{O}_{2}$ formation during metal-catalyzed glycoxidation reactions $(26,27)$. In other work $(20,21)$, we have shown that the increase in glycoxidation products during glycation of collagen under oxidative conditions is accompanied by the formation of fluorescent products and the cross-linking of collagen. Similar changes in levels of glycoxidation products, AGE-like fluorescence and cross-linking occur naturally with age in collagen in vivo, and we demonstrate here that these changes are also accompanied by age-dependent increases in both MetSO and $o$-Tyr in human skin collagen. Partial correlations, independent of age, between levels of MetSO and $o$-Tyr and levels of CML and pentosidine in collagen were statistically significant in nondiabetic subjects (Table II), suggesting that levels of both oxidation and glycoxidation products were affected by
Table I. Correlations between Oxidation and Glycoxidation Products in Human Skin Collagen

\begin{tabular}{cccccc}
\hline & \multicolumn{2}{c}{ CML } & & \multicolumn{2}{c}{ Pentosidine } \\
\cline { 2 - 3 } \cline { 5 - 6 } & Nondiabetic & Diabetic & & Nondiabetic & Diabetic \\
\hline -Tyr & & & & 0.81 \\
$r$ & 0.82 & 0.72 & & 0.68 & 0.0001 \\
$P$ & $<0.0001$ & 0.004 & & 0.008 & 14 \\
$n$ & 21 & 14 & & 18 & 0.84 \\
MetSO & & & & 0.85 & 0.001 \\
$r$ & 0.73 & 0.82 & & 0.0003 & 11 \\
$P$ & 0.0007 & 0.007 & & 13 & \\
\hline
\end{tabular}

a similar background level or set point of oxidative stress. The loss of partial correlations between oxidation and glycoxidation products in the diabetic population is consistent with a dissociation between rates of oxidation and glycoxidation in these patients, possibly resulting from effects of variations in glycemic control, protein glycation, and duration of disease on the rate of formation of glycoxidation products among diabetic patients.

The significant partial correlations between levels of amino acid oxidation and glycoxidation products in skin collagen from nondiabetic subjects and the loss of this correlation in the collagen from diabetic patients (Table II), and the observation that age-corrected levels of MetSO and $o$-Tyr in skin collagen were not increased in diabetic compared with nondiabetic subjects (Figs. 4 and 5) argue strongly that there is not a systemic or generalized increase in oxidative stress in diabetes, at least in the extracellular compartment. These results support the conclusion of Dyer et al. (7) that the increase in glycoxidation products in human skin collagen in diabetes can be explained by the increase in glycemia during the course of diabetes, without invoking a diabetes-dependent increase in oxidative stress. Dyer et al. (7) also proposed, however, that patients with comparable levels of hyperglycemia and duration of diabetes, but with higher set points for oxidative stress, may be at increased risk for development of complications. Like hereditary suscep-

Table II. Age-corrected Correlations between Oxidation and Glycoxidation Products in Human Skin Collagen

\begin{tabular}{llll}
\hline \multicolumn{1}{c}{ Correlated products } & Nondiabetic & Nondiabetic & Diabetic \\
\hline & $0-82 y r$ & $17-58 y r$ & $17-58 y r$ \\
$o$-Tyr $\leftrightarrow$ CML & $r=0.55$ & $r=0.53$ & $r=0.11$ \\
& $P=0.01$ & $P=0.08$ & $P=0.74$ \\
& $n=21$ & $n=13$ & $n=14$ \\
-Tyr $\leftrightarrow$ pentosidine & $r=0.58$ & $r=0.60$ & $r=0.16$ \\
& $P=0.01$ & $P=0.054$ & $P=0.61$ \\
MetSO $\leftrightarrow$ CML & $n=18$ & $n=13$ & $n=14$ \\
& $r=0.54$ & $r=0.53$ & $r=0.16$ \\
MetSO pentosidine & $P=0.03$ & $P=0.14$ & $P=0.71$ \\
& $n=17$ & $n=10$ & $n=9$ \\
& $r=0.72$ & $r=0.77$ & $r=0.48$ \\
& $P=0.009$ & $P=0.03$ & $P=0.16$ \\
& $n=13$ & $n=9$ & $n=11$ \\
\hline
\end{tabular}


tibility to hypertension or hyperlipidemia, differences in genetically determined set points for oxidative stress may still be an important factor determining individual risk for development of complications. These issues could not be addressed in the present study because of the limited number of samples available and the range of complications status among the patients studied, but will be addressed in future research.

Although we have concluded from our studies that oxidative stress is not generally increased in the extracellular milieu in diabetes, there is considerable experimental and clinical evidence implicating increased oxidative stress in the development of diabetic complications (reviewed in references 1, 8-10, 28-33). For example, antioxidant vitamins, drugs, and thiol compounds have retarded the development of retinopathy (34), neuropathy $(35,36)$, and vasculopathy $(37)$ in the streptozotocin-induced diabetic rat model. However, the diabetic rat is frequently a severe, chronically ketoacidotic model for the study of diabetic complications, and these animals commonly fail to increase in weight or even suffer severe weight loss as a result of muscle wasting during the course of experiments. Observations made in this model may not always be relevant to understanding the pathogenesis of complications developing more gradually in less extreme human diabetes (38). On the other hand, deficits in ascorbate and glutathione homeostasis are well documented in human diabetes (reviewed in references $1,28,30$ ), suggesting that, independent of observations in animal models, antioxidant defenses may be compromised in human diabetes. These apparent contradictions are not readily resolved, but it should be recognized that increases in oxidative stress may be associated with the complications and tissue damage, rather than with diabetes per se. Thus, levels of AGEs in collagen (7) and in plasma are significantly higher in diabetic patients with renal disease, compared with those with normal renal function, but are also increased in nondiabetic patients with renal disease $(39,40)$, suggesting that nephropathy, rather than diabetes, may be the more significant determinant of oxidative stress and the rate of glycoxidation reactions in vivo.

Threshold effects may also be important in explaining why oxidative stress is not increased in diabetes; i.e., even with measurable decreases in antioxidant defenses in human diabetes, these defenses may still be sufficient to limit increased glucose or lipid dependent oxidative damage to tissue proteins. Thus, the increase in glycoxidation products (CML and pentosidine), but not oxidation products (MetSO and $o$-Tyr), in diabetes suggests that oxidative defenses are adequate-that the threshold has not been exceeded. The increase in glycoxidation products, and possibly similar products derived from lipid peroxidation, may be viewed as the result of increased levels of oxidizable substrates (i.e., carbohydrates or lipids) rather than increased background levels of ROS. In the scheme shown in Fig. 6, oxidative stress may be represented by the steady state level of ROS $\left(\left[\mathrm{O}_{2}\right]^{*}\right)$, the balance between the rates of generation $\left(\mathrm{k}_{1}\right)$ and detoxification $\left(\mathrm{k}_{2}\right)$ of reactive oxygen. An increase in an oxidizable substrate, such as glucose, may lead to increased substrate-dependent oxidative damage (glycoxidation products) with a substrate-specific rate constant $\left(\mathrm{k}_{3}\right)$. The substrate-dependent increase in glycoxidation of collagen in diabetes could occur without a change in oxidative stress $\left[\mathrm{O}_{2}\right]^{*}$, and the rate of oxidative modification of the protein backbone, which is dependent on $\left[\mathrm{O}_{2}\right]^{*}$ and assessed by levels of MetSO and $o$-Tyr, would not be altered.

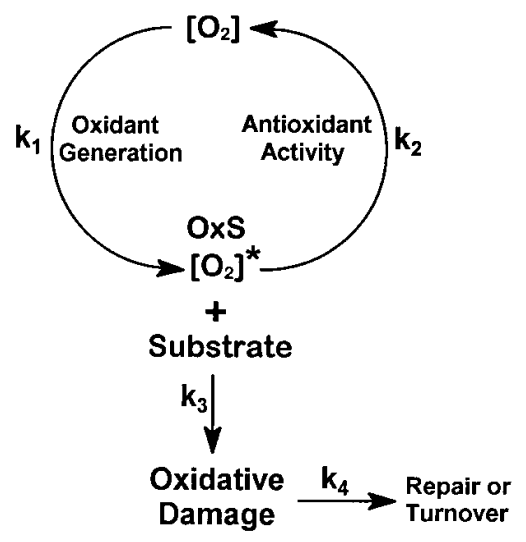

Figure 6. Relationship between rates of oxidant generation, antioxidant activity, oxidative stress $(\mathrm{OxS})$, and oxidative damage. $\left[\mathrm{O}_{2}\right]^{*}$ represents various forms of reactive oxygen (e.g., hydroxyl radical, superoxide, and hydroperoxy radical, or metal-oxygen complexes) generated with an overall rate constant $\mathrm{k}_{1}$ and detoxified with an overall rate constant $\mathrm{k}_{2}$ by a variety

of enzymatic and nonenzymatic mechanisms. Oxidative stress $(O x S)$ may be considered a measure of the steady state level of $\left[\mathrm{O}_{2}\right]^{*}$. Reaction of $\left[\mathrm{O}_{2}\right]^{*}$ with a variety of biomolecule substrates, such as carbohydrates, lipids, proteins, and nucleic acids, with varying rate constants $\mathrm{k}_{3}$ depending on the substrate, leads to formation of oxidation products in tissues. The overall rate of formation of oxidation products (Oxidative Damage) depends on ambient levels of both substrate and $\left[\mathrm{O}_{2}\right]^{*}$. Oxidatively damaged proteins may also be catabolized and replaced during turnover processes $\left(\mathrm{k}_{4}\right)$, thus the steady state level of oxidative damage in tissues is affected by a range of substrate concentrations and rate constants.

A limitation of our studies is the focus on skin collagen, which may not be an appropriate sensor for intracellular oxidative stress in other tissues. Indeed, skin is not a major site of pathology in diabetes, and collagen is an extracellular matrix protein. It is possible that oxidative stress may be increased specifically within cells, for example, as a result of altered glucose metabolism during hyperglycemia (reviewed in references $10,28,41)$, by the action of AGEs bound to cell surface receptors (42), or by other mechanisms and that there may be tissue-specific variations in levels of antioxidant vitamins and enzymes, glutathione, and other cofactors $(43,44)$. However, there are strong correlations between levels of glycoxidation products in skin collagen and the severity of diabetic retinal, renal, and vascular disease (3-5), and cellular membranes are freely permeable to ROS, such as $\mathrm{O}_{2} \mathrm{H}^{\bullet}$ (the protonated form of superoxide, $\mathrm{pKa} \sim 4.5$ ) and $\mathrm{H}_{2} \mathrm{O}_{2}$. Resolution of these problems will require studies of other indicators of oxidative stress in diabetes, such as protein carbonyls (45), lipid peroxidation products $(14,46)$, and amino acid oxidation products in cellular proteins. A more comprehensive study of age-adjusted levels of MetSO and $o$-Tyr in skin collagen from diabetic patients with similar age of onset, duration of disease, and long-term glycemic control, but with significant differences in status of complications, should provide a clearer insight into the relationship between oxidative stress and the development of diabetic complications.

Oxidative stress and aging. In addition to identification of MetSO and $o$-Tyr as indicators of oxidative damage to tissue proteins, our results provide chemical evidence, consistent with the free radical theory of aging (47). According to this theory, aging is the result of cumulative oxidative damage to biomolecules. The increase in glycoxidation products and amino acid oxidation products in skin collagen provides evidence for an age-dependent increase in oxidative damage, al- 
though it does not establish a causal relationship between oxidative stress and aging. The relationship between levels of amino acid oxidation products in skin collagen and species life span is also unknown. However, these issues can be addressed experimentally by measuring the rates of increase in MetSO and $o$-Tyr in collagen from species of varying life spans and by evaluating the effects of caloric restriction, which extends maximum life span in numerous species (48), on the rate of increase in MetSO and $o$-Tyr in collagen. Measurements of these relatively stable amino acid oxidation products should provide a direct indicator of oxidative damage and would not be compromised by changes in glycemia and protein glycation.

\section{Acknowledgments}

The authors thank Dr. Davis F. Gates, Department of Biometry, Medical University of South Carolina, for assistance with statistical analyses.

This work was supported by research grants DK-19971 to J.W. Baynes from the National Institutes of Diabetes, Digestive, and Kidney Diseases, and AG-11472 to S.R. Thorpe from the National Institute of Aging.

\section{References}

1. Baynes, J.W. 1991. The role of oxidative stress in the development of complications in diabetes. Diabetes. 40:405-412.

2. Vlassara, H., R. Bucala, and L. Striker. 1994. Pathogenic effects of advanced glycosylation: biochemical, biologic and clinical implications for diabetes and aging. Lab. Invest. 70:138-151.

3. Sell, D.R., A. Lapolla, P. Odetti, J. Fogarty, and V.M. Monnier. 1992. Pentosidine formation in skin correlates with severity of complications in individuals with long-standing IDDM. Diabetes. 41:1286-1292.

4. McCance, D.R., D.G. Dyer, J.A. Dunn, K.E. Bailie, S.R. Thorpe, J.W. Baynes, and T.J. Lyons. 1993. Maillard reaction products and their relation to the complications of diabetes J. Clin. Invest. 91:2470-2478.

5. Beisswenger, P.J., L.L. Moore, T. Brinck-Johnsen, and T.J. Curphey. 1993. Increased collagen-linked pentosidine levels and advanced glycosylation end products in early diabetic nephropathy. J. Clin. Invest. 92:212-217.

6. Sell, D.R., and V.M. Monnier. 1990. End-stage renal disease and diabetes catalyze the formation of a pentose-derived crosslink from aging human collagen. J. Clin. Invest. 75:380-384.

7. Dyer, D.G., J.A. Dunn, S.R. Thorpe, K.E. Bailie, T.J. Lyons, D.R. McCance, and J.W. Baynes. 1993. Accumulation of Maillard reaction products in skin collagen in diabetes and aging. J. Clin. Invest. 91:2463-2469.

8. Wolff, S.P., and R.T. Dean. 1987. Glucose autoxidation and protein modification: the potential role of autoxidative glycosylation in diabetes. Biochem. J. 245:243-250.

9. Wolff, S.P., Z.Y. Jiang, and J.V. Hunt. 1991. Protein glycation and oxidative stress in diabetes mellitus and ageing. Free Radical Biol. Med. 10:339-352.

10. Baynes, J.W. 1996. The role of oxidation in the Maillard reaction in vivo. In The Maillard Reaction: Consequences for the Chemical and Life Sciences. R. Ikan, editor. John Wiley \& Sons Inc., New York. pp. 55-72.

11. Baynes, J.W., and S.R. Thorpe. 1996. The role of oxidative stress in diabetic complications. Curr. Opin. Endocrinol. Diabetes. 3:277-284.

12. Grandhee, S.K., and V.M. Monnier. 1991. Mechanism of formation of the Maillard protein cross-link, pentosidine: glucose, fructose and ascorbate as pentosidine precursors. J. Biol. Chem. 266:11649-11653.

13. Dyer, D.G., J.A. Blackledge, S.R. Thorpe, and J.W. Baynes. 1991. Formation of pentosidine during nonenzymatic browning of proteins by glucose: identification of glucose and other carbohydrates as possible precursors of pentosidine in vivo. J. Biol. Chem. 266:11654-11660.

14. Fu, M.-X., J.R. Requena, A.J. Jenkins, T.J. Lyons, J.W. Baynes, and S.R. Thorpe. 1995. The advanced glycation end product, $\mathrm{N}^{\epsilon}$-(carboxymethyl)lysine, is a product of both lipid peroxidation and glycoxidation reactions. J. Biol. Chem. 271:9982-9986.

15. Brot, N., and H. Weissbach. 1983. Biochemistry and physiological role of methionine sulfoxide residues in proteins. Arch. Biochem. Biophys. 223:271-281.

16. Vogt, W. 1995. Oxidation of methionine residues in proteins: tools, targets, and reversal. Free Radical Biol. Med. 18:93-105.

17. Huggins, T.G., M.C. Wells-Knecht, N.A., Detorie, J.W. Baynes, and S.R. Thorpe. 1993. Formation of $o$-tyrosine and dityrosine in protein during radiolytic and metal-catalyzed oxidation. J. Biol. Chem. 268:12341-12347.

18. Wells-Knecht, M.C., T.G. Huggins, D.G. Dyer, S.R. Thorpe, and J.W.
Baynes. 1993. Oxidized amino acids in lens proteins with age. Measurement of $o$-tyrosine and dityrosine in the aging human lens. J. Biol. Chem. 268:1234812352.

19. Knecht, K.J., J.A. Dunn, K.F. McFarland, S.R. Thorpe, and J.W. Baynes. 1991. Oxidative degradation of glycated proteins: effect of diabetes and aging on carboxymethyllysine levels in human urine. Diabetes. 40:190-196.

20. Fu, M.-X., K.J. Knecht, S.R. Thorpe, and J.W. Baynes. 1992. Role of oxygen in the crosslinking and chemical modification of protein by glucose. Diabetes. 41(Suppl. 2):42-48.

21. Fu, M.-X., K.J. Wells-Knecht, J.A. Blackledge, T.J. Lyons, S.R. Thorpe, and J.W. Baynes. 1994. Glycation, glycoxidation and crosslinking of collagen by glucose: kinetics, mechanisms and inhibition of late stages of the Maillard reaction. Diabetes. 43:676-683.

22. Wells-Knecht, M.C., S.R. Thorpe, and J.W. Baynes. 1995. Pathways of formation of glycoxidation products during glycation of collagen. Biochemistry. 34:15134-15141.

23. Watkins, N.G., C.I. Neglia, D.G. Dyer, S.R. Thorpe, and J.W. Baynes. 1987. Effects of phosphate on the kinetics and specificity of glycation of protein. J. Biol. Chem. 262:7207-7212.

24. Zyzak, D.V., J.M. Richardson, S.R. Thorpe, and J.W. Baynes. 1995. Formation of reactive intermediates from Amadori compounds under physiological conditions. Arch. Biochem. Biophys. 316:547-554.

25. Finot, P.A., and D.E. Furniss. 1986. Nephrocytomegaly in rats induced by Maillard reaction products: the involvement of metal ions. Dev. Food Sci. 13: 493-502.

26. Jiang, Z.Y., A.C.S. Woolard, and S.P. Wolff. 1990. Hydrogen peroxide generation during experimental protein glycation. FEBS Lett. 268:69-71.

27. Elgawish, A., M. Glomb, M. Friedlander, and V.M. Monnier. 1996. Involvement of hydrogen peroxide in collagen cross-linking by high glucose in vitro and in vivo. J. Biol. Chem. 271:12964-12971.

28. Williamson, J.R., K. Chang, M.F. Khalid, K.S. Hasan, Y. Ido, T. Kawamura, J.R. Nyengaard, M. Van den Enden, C. Kilo, and R.G. Tilton. 1993. Hyperglycemic pseudohypoxia and diabetic complications. Diabetes. 42:801-813.

29. Cameron, N.E., and M.A. Cotter. 1993. Potential therapeutic approaches to the treatment or prevention of diabetic neuropathy: evidence from experimental studies. Diabetic Medicine. 10:593-605.

30. Baynes J.W. 1995. Reactive oxygen in the aetiology and complications of diabetes. In Drugs, Diet and Disease. Vol. 2: Mechanistic Approaches to Diabetes. C. Ioannides, editor. Pergamon Press, London. 203-240.

31. Giugliano, D., A. Ceriello, and G. Paolisso. 1996. Oxidative stress and diabetic vascular complications. Diabetes Care. 19:257-265.

32. Lyons, T.J., and A.J. Jenkins. 1996. Glycation, oxidation, and lipoxidation in the development of the complications of diabetes mellitus: a carbonyl stress hypothesis. Diab. Rev. In press.

33. Van Dam, P.S., B.S. Van Asbeck, D.W. Erkelens, J.J. M. Marx, W.H. Gispen, and B. Bravenboer. 1996. The role of oxidative stress in neuropathy and other diabetic complications. Diabetes Metab. Rev. 11:181-192.

34. Kunisaki, M., S.E. Bursell, A.C. Clermont, H. Ishii, L.M. Ballas, M.R. Jirousek, F. Umeda, H. Nawata, and G.L. King. 1995. Vitamin E prevents diabetes-induced abnormal retinal blood flow via the diacylglycerol-protein kinase C pathway. Am. J. Physiol. 269:E239-E246.

35. Cameron, M.A., A. Love, M.J. Watt, N.E. Cameron, and K.C. Dines. 1995. Effects of natural free radical scavengers on peripheral nerve and neurovascular function in diabetic rats. Diabetologia. 38:1285-1294.

36. Sagara, M., J. Satoh, R. Wada, S. Yagihashi, K. Takahashi, M. Fukuzawa, G. Muto, Y. Muto, and T. Toyota. 1996. Inhibition of development of peripheral neuropathy in streptozotocin-induced diabetic rats with $\mathrm{N}$-acetylcysteine. Diabetologia. 39:263-269.

37. Keegan, A., H. Walbank, M.A. Cotter, and N.E. Cameron. 1995. Chronic vitamin E treatment prevents defective endothelium-dependent relaxation in diabetic rat aorta. Diabetologia. 38:1475-1478.

38. Requena, J.R., and J.W. Baynes. 1997. Studies in animal models on the role of glycation and advanced glycation end-products (AGEs) in the pathogenesis of diabetic complications: pitfalls and limitations. In Lessons from Animal Diabetes. Vol. VII. A. Sima, editor. Birkhäuser, Boston. In press.

39. Makita, Z., S. Radoff, E.J. Rayfield, Z. Yang, E. Skolnik, V. Delaney, E.A. Friedman, A. Cerami and H. Vlassara. 1991. Advanced glycosylation end products in patients with diabetic nephropathy. N. Engl. J. Med. 325:836-842.

40. Papastanasiou, P., L. Grass, H. Rodela, A. Patrikarea, D. Oreopoulos, and E.P. Diamandis. 1994. Immunological quantification of advanced glycosylation end-products in the serum of patients on hemodialysis or CAPD. Kidney Int. 46:216-222.

41. Ceriello, A., P. dello Russo, P. Armstad, and P. Cerutti. 1996. High glucose induces antioxidant enzymes in human endothelial cells in culture: evidence linking hyperglycemia and oxidative stress. Diabetes. 45:471-477.

42. Schmidt, A.M., O. Hori, J. Brett, S.-D. Yan, J.L. Wautier, and D. Stern. 1994. Cellular receptors for advanced glycation end products: implications for induction of oxidant stress and cellular dysfunction in the pathogenesis of vascular lesions. Arterioscler. Thromb. 14:1521-1528.

43. Godin, D.V., and S.A. Wohaieb. 1988. Reactive oxygen processes in diabetes. In Oxygen Radicals in Pathophysiology of Heart Disease. P.K. Singal, editor. Kluwer Academic Publishers, Norwell, MA. pp. 302-322. 
44. Oberley, L.W. 1988. Free radicals and diabetes. Free Radical Biol. Med. 5:113-124.

45. Stadtman, E.R. 1995. Role of oxidized amino acids in protein breakdown and stability. Methods Enzymol. 258:379-393.

46. Requena, J.R., M.-X. Fu, M.U. Ahmed, A.J. Jenkins, T.J. Lyons, J.W. Baynes, and S.R. Thorpe. 1997. Quantitation of malondialdehyde and 4-hydroxynonenal adducts to lysine residues in native and oxidized human low-density li- poprotein. Biochem J. 322:317-325.

47. Harman, D. 1992. Role of free radicals in aging and disease. Ann. NY Acad. Sci. 673:126-141.

48. Weindruch, R., and R.L. Walford. 1988. The Retardation of Aging and Disease by Dietary Restriction. Charles C. Thomas, Publisher, Springfield, IL. $436 \mathrm{pp}$. 